\title{
Cytoplasmic Expression of DCLK1-S, a Novel DCLK1 Isoform, Is Associated with Tumor Aggressiveness and Worse Disease-Specific Survival in Colorectal Cancer
}

\author{
Elham Kalantari \\ Iran University of Medical Sciences \\ Roya Ghods \\ Iran University of Medical Sciences \\ Leili Saeednejad Zanjani \\ Iran University of Medical Sciences \\ Mandana Rahimi \\ Iran University of Medical Sciences \\ Leila Eini \\ Iran University of Medical Sciences \\ Mahdieh Razmi \\ Iran University of Medical Sciences \\ Mohsen Asadi-Lari \\ Iran University of Medical Sciences \\ Zahra Madjd ( $\sim$ majdjabari.z@iums.ac.ir) \\ Iran University of Medical Sciences https://orcid.org/0000-0001-7329-2583
}

\section{Primary research}

Keywords: Colorectal cancer, DCLK1-S, Polyclonal antibody, Immunohistochemistry, Tissue microarray

Posted Date: March 19th, 2021

DOI: https://doi.org/10.21203/rs.3.rs-148922/v2

License: (1) This work is licensed under a Creative Commons Attribution 4.0 International License. Read Full License

Version of Record: A version of this preprint was published at Cancer Biomarkers on December 23rd, 2021. See the published version at https://doi.org/10.3233/CBM-210330. 


\section{Abstract}

Background: Oncogenic role of doublecortin-like kinase 1 (DCLK1) as a putative cancer stem cell (CSC) marker has been clarified in colorectal cancer (CRC). Isoform-specific function of DCLK1 has highlighted key role of the DCLK1-S (short isoform) in tumor maintenance, progression, and invasion. Considering the lack of commercial anti-DCLK1-S antibody suitable for immunohistochemical (IHC) application, this study was conducted to produce and validate an anti-DCLK1-S polyclonal antibody in order to specifically evaluate expression pattern and clinical significance of short isoform of DCLK1 in colorectal cancer tissues.

Methods: Rabbit immunization was performed against a synthetic peptide corresponding to the published six specific amino acid sequences of DCLK1-S, and production of antibody was evaluated by enzyme-linked immunosorbent assay (ELISA). After IHC assessment of the purified anti-DCLK1-S polyclonal antibody, it was used to undertake a definitive study for determining prognostic significance of DCLK1-S expression in a well-defined tissue microarray (TMA) series including 348 CRC and 51 adjacent normal tissues with a follow-up period of 108 months.

Results: Positive immunoreactivity of DCLK1-S was found in $84.2 \%$ of CRC samples. Cytoplasmic expression was the main localization of DCLK1-S compared to nuclear and membranous area of tumor cells. Expression of DCLK1-S in CRC samples was significantly higher compared to adjacent normal samples $(P<0.001)$. A positive significant association was found between high cytoplasmic expression of DCLK1-S and advanced tumor, nodes, and metastases $(T N M)$ stage $(P<0.001)$ as well as the increased tumor differentiation $(P=0.02)$. Moreover, the patients with CRC whose tumors showed higher cytoplasmic expression of DCLK1-S had worse disease-specific survival (DSS) (log-rank test, $P=0.03$ ) and 5-year DSS rate $(P=0.01)$. Additionally, the improved prognostic value was seen in the patients with $C R C$ with high DCLK1-S expression versus moderate expression (HR: 2.70, 95\% Cl: $0.98-7.38 ; p=0.04$ ) by multivariate analysis.

Conclusions: Our findings strongly supported that DCLK1-S isoform may play a crucial role in invasion, tumor aggressive behavior, and worsened DSS of the patients with CRC. Importantly, high cytoplasmic expression of DCLK1-S compared to moderate expression could be considered as an independent prognostic factor influencing DSS. Taken together, DCLK1-S can be a candidate as a promising prognostic and targeted-therapeutic indicator for effective treatment of CRC.

\section{Background}

Colorectal cancer (CRC) is the fourth most frequently diagnosed cancer and leading cause of global death [1]. Despite developments in anti-cancer treatments, most CRC cases are resistant to conventional cancer therapies. Surgery, radiotherapy, chemotherapy, and targeted therapy are used as the current treatment for the patients with CRC. Combining these traditional methods with new targeted-therapy strategies could increase survival rate of the patients with CRC [2]. Therefore, identifying biomarkers can be considered an essential step for improving targeted molecular therapies (immunotherapy) and increasing survival of the patients with cancer. Considering crucial role of cancer stem cells (CSCs) in tumorigenesis, tumor invasion, and drug-resistance, many efforts have been made to discover specific markers in order to target CSCs in various cancers including CRC [3-6]. Among various markers used for identification of CRC-CSCs, doublecortin-like kinase 1 (DCLK1), as a putative CSC marker has been shown to have a crucial oncogenic role in various in-vivo and in-vitro experiments, particularly in pancreatic and colon cancers $[5,7-10]$ so that, DCLK1 is called as a well-known CRC-CSC marker distinguished cancer cells from normal cells in Apc Min/+ mice [7].

In the recent decades, many experiments have focused on biological function of two different transcripts of DCLK1; long isoform (DCLK1-L, NM_004734.4) and short isoform (DCLK1-S, NM_001195415.1) with molecular weight of $~ 80-82$ and $\sim 45-50$ KDa, respectively [9, 11, 12]. Evidence has suggested more than $98 \%$ of similarity between DCLK1-L and DCLK1-S isoforms at C-terminal end of both proteins, while the only difference in the sequence of six amino acids was found at N-terminal end of the DCLK1-S protein $[9,10]$. Further molecular evidence regarding isoform-specific function of DCLK1 in tumor initiation, proliferation, and aggressiveness has shown oncogenic role of DCLK1-L at the early stages and DCLK1-S in progression and invasion of CRC and pancreatic cancer $[8,13,14]$. Epigenetically, due to silencing of $5^{\prime}(\mathrm{a})$ promoter of DCLK1 gene as an early event during CRC carcinogenesis, overexpression of DCLK1 in CRC experiments was a vague issue [9]. This controversy was addressed when oncogenic biological role of an alternative transcriptional $\beta$-promoter of DCLK1 was clarified [9]. O'Connell et al., suggested that although DCLK1-S isoform originated from $\beta$-promoter may have the primary CSC characteristics of DCLK1 protein, DCLK1-L could play a leading role in identification of normal or low-stage vs. high-stage CRC cells $[9,15]$. They showed higher expression of DCLK1-S in the patients with CRC with shorter overall survival by quantitative real-time polymerase chain reaction (qRT-PCR) technique and ultimately, they suggested DCLK1-S as a specific CRC biomarker for characterization of high-risk patients after colonoscopy $[9,10]$. Other molecular experiments have reported overexpression of DCLK1-S vs. DCLK1-L in tumor cells compared to normal cells influenced by forkhead-box-D3 (FOXD3) gene in regulation of DCLK1-S in colorectal carcinomas [16], although more investigations are required to define specific biological difference of these two isoforms yet. 
In our previous research, expression pattern and prognostic significance of DCLK1 have been studied in colorectal, gastric, and bladder carcinomas [17-21] using available commercial anti-DCLK1 antibodies that can detect C-terminal domain of both DCLK1-L/S isoforms or only N-terminal end of DCLK1-L isoform, while role of the main isoform to overexpress DCLK1 has remained unknown. To the best of our knowledge, there is no commercial anti-DCLK1-S antibody, and also no study has been previously performed to investigate prognostic significance of DCLK1-S on tumor tissues, particularly in CRC by immunohistochemistry (IHC). Therefore, for addressing these gaps, the present study was conducted to generate an anti-DCLK1-S-specific polyclonal antibody elicited by six amino acids $₫$ synthetic peptide that has been reported as the only different form in the sequence homology epitopes of both DCLK1 isoforms (DCLK1-L and DCLK1-S)[10].

In the current study, for the first time, membranous, cytoplasmic, and nuclear localization of DCLK1-S elicited by polyclonal anti-DCLK1-S antibody, generated in our laboratory was evaluated in a large number of formalin-fixed, paraffin-embedded (FFPE) series of CRC samples by tissue microarray (TMA)-based IHC analysis. Prognostic significance of short isoform of DCLK1 (DCLK1-S) was also investigated in this series of the patients with CRC.

\section{Methods}

\section{Production of anti-DCLK1-S-specific polyclonal antibody}

Polyclonal antibody to DCLK1-S was generated in our laboratory, as described previously [22-24]. A synthetic peptide corresponding to the published amino acid sequence of DCLK1-S isoform (MLELIE) was selected as immunogen [10]. A cysteine residue was added to Cterminal of peptide (NH2-Met-Leu-Glu-Leu-Ile-Glu-Cys-COOH) for conjugation with Imject Maleimide-activated mckeyhole limpet hemocyanin (KLH) (Thermo Scientific, Rockford, IL, USA) and used for immunization of two female New Zealand White Rabbits purchased from Pasteur Institute of Iran. To assess conjugation efficacy, the peptide was also conjugated with Imject Maleimide-activated bovine serum albumin (BSA) (Thermo Scientific, Rockford, IL, USA). Conjugation efficacy was investigated by analysis of electrophoretic pattern of the BSA-conjugate [22]. Production of DCLK1-S specific antibody was evaluated by enzyme-linked immunosorbent assay (ELISA) [25]. AntiDCLK1-S specific antibodies were purified by the peptide coupled to CNBr-activated Sepharose-4B. Purity and reactivity of the purified antibodies were assessed by sodium dodecyl-sulfate polyacrylamide gel electrophoresis (SDS-PAGE) and ELISA techniques, respectively. Immunoreactivity pattern of the generated antibody was compared with available commercial anti-DCLK1 antibody; ab31704 (which is commonly used to detect long and short DCLK1 isoforms; Abcam, UK) in CRC tissues by IHC method.

\section{Indirect ELISA}

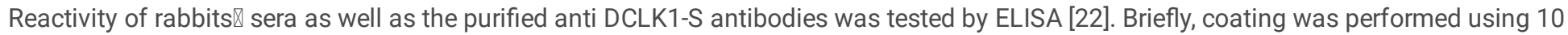
$\mu \mathrm{g} / \mathrm{ml}$ of the peptide diluted in phosphate-buffered saline (PBS, $0.15 \mathrm{M}, \mathrm{pH} 7.2$ ), which was incubated at $37 \circ \mathrm{C}$ for $1 \mathrm{~h}$ followed by overnight incubation at $4 \circ \mathrm{C}$. After several washes, plates were blocked using $2.5 \%$ skimmed milk at $37 \circ \mathrm{C}$ for $1 \mathrm{~h}$ and then, serial dilutions of rabbits $\mathbb{B}$ sera or the purified antibodies were added. Following serum incubation ( $37^{\circ} \mathrm{C}$ for $1.5 \mathrm{~h}$ ) and washing, a horseradish peroxidase (HRP) conjugate of sheep anti-rabbit immunoglobulin (Ig) antibody (Avicenna Research Institute, Tehran, Iran) diluted 1:1500 in phosphate buffered saline-Tween 20 (PBS-T ) was added to the wells $\left(37^{\circ} \mathrm{C}, 1.5 \mathrm{~h}\right)$. Following washing, substrate, 3,3',5,5'-tetramethylbenzidine (TMB) (Pishtaz Teb Zaman, Iran) was added and optical density (OD) values were measured at wavelength of $450 \mathrm{~nm}$ on a microplate reader (Synergy HTXMulti-Mode Reader, BioTek, USA).

\section{Sample collection and patients characteristics}

FFPE series of 385 CRC samples and 70 matched adjacent normal tissues were collected from university hospitals including Hasheminejad, Rasool Akram, and Firoozgar during 2010 - 2019 in Tehran, Iran. Clinicopathological information including age, gender, tumor size, tumor differentiation, vascular and neural invasion, lymph node involvement, tumor, nodes, and metastases (TNM) staging, distant metastasis, and tumor recurrence was collected from the patients' documents. None of the patients with CRC in this study had received neoadjuvant treatment before surgery. Size cutoff and TNM stage of the tumors were determined according to the American joint committee on cancer/ union for international cancer control (AJCC/UICC) and TNM staging system, respectively [26, 27]. The patients' data were kept anonymous in all steps of the study. Interval from the date of surgery to the date of death by CRC was used for diseasespecific survival (DSS) analysis. Moreover, progression-free survival (PFS) was defined as the interval between the primary surgery and the last follow-up visit if the patient showed no evidence of disease, tumor recurrence, or distant CRC metastasis. The current study was approved by the Human Research Ethics Committee of the Iran University of Medical Sciences, Tehran, Iran (Ref No: IR.IUMS.REC 1396.32186). All the procedures performed in this study were in accordance with the 1964 Declaration of Helsinki and its later amendments. Informed consent was obtained from all individual participants, parents or legally authorized representatives of participants at the time of sample collection with routine consent forms. 
Colorectal tissue arrays were prepared and constructed by a TMA instrument (Minicore; ALPHELYS, Plaisir, France), as described previously $[4,28,29]$. Three representative areas of the tumors were pointed on hematoxylin \& eosin ( $\mathrm{H} \& \mathrm{E})$ slides of CRC samples by pathologist. Different regions of each tumor were punched into TMA recipient blocks in three copies. Based on TMA studies, each core would denote to the whole tissue's staining pattern with $90 \%$ of accuracy, while using two cores or more could increase this accuracy by $95-99 \%$, respectively [30]. In this study, for overcoming heterogeneity in antigen expression and increasing accuracy and validity of the analysis, three cores were evaluated from each tissue sample. Mean score of the three cores was calculated as the final score for IHC analysis of DCLK1-S.

\section{Immunohistochemistry}

Immunostaining of CRC slides (whole tissue and TMA slides) was performed by standard chain polymer-conjugated antibody (EnVision), as described previously $[4,19,31]$. Briefly, following de-waxing at $60^{\circ} \mathrm{C}$ for $30 \mathrm{~min}$ and rehydration steps, heat-activated antigen was retrieved by autoclaving the sections in citrate buffer $(10 \mathrm{mM}, \mathrm{pH} 6)$ at $95^{\circ} \mathrm{C}$ for $11 \mathrm{~min}$. Endogenous peroxidase activity and non-specific binding sites were blocked by $\mathrm{H} 2 \mathrm{O} 2(3 \%)$ and $5 \%$ normal sheep serum diluted in protein block (Dako, CA, USA), respectively. The sections were incubated overnight using a polyclonal anti-DCLK1-S antibody generated in our laboratory $(0.75 \mu \mathrm{g} / \mathrm{ml})$ at $4{ }^{\circ} \mathrm{C}$. Then, the sections were treated with the secondary antibody, ${ }^{\text {TM}}$ Mouse/Rabbit PolyVue HRP/DAB Detection kit (standard EnVision-HRP kit, Bio pharmadx), at room temperature (RT). The 3; 3'-diaminobenzidine (DAB) substrate as a chromogen was used to visualize immune signals. The sections were counterstained with hematoxylin for $5 \mathrm{~min}$, were dehydrated and finally, were mounted. Humans $\mathbb{C R C}$ whole tissue was selected as positive control for DCLK1-S. In negative reagent controls, the primary antibody was replaced by non-immune rabbit IgG as isotype control to confirm specific bindings of anti-DCLK1-S antibody. In addition, in all the experiments, specificity of the produced antibodies was evaluated by blocking antibodies with saturating concentration (1:100 molar ratio) of immunizing peptide before incubating the slides with the primary antibody. The primary antibody was also replaced by tris buffer saline (TBS, pH: 7.4) as negative control to confirm the absence of non-specific bindings of the secondary antibody. Normal and cancerous tissues of ovary [32], testis, and skin were also used as negative control tissues based on protein atlas databases for further confirmation of specificity of our polyclonal antibody. Digital images were captured by an H550S microscope (Nikon, Japan) and a Digital sight DS-LS camera (Nikon, Japan).

\section{Evaluation of immunostaining}

Scoring step was performed semi-quantitatively in a coded manner, by two pathologists (MA and MR) who were blinded to clinical and pathological parameters (as described previously) [33], and a consensus agreement was achieved. Given an agreement to the final score of DCLK1-S expression, the general distribution of the tumor cells was assessed at 10x magnification. Then, positive cells were evaluated semi-quantitatively at higher magnifications, and final scores were given, respectively. Percentage of positive cells, intensity of the staining, and histochemical score (H-score) were three scoring methods by which expression pattern of DCLK1-S was evaluated. Intensity of immunostaining was divided into four groups; 0 (negative), 1 (weak), 2(moderate), and 3(strong) staining. Percentage of positive cells was valued semi-quantitatively and was scored as $0-100 \%$. The $H$-score was obtained by multiplying intensity $(0-3)$ by percentage scores $(0-$ $100 \%$ ), which led to generation of the final scores of 0-300. In the present study, H-score was divided into three groups: 0-100 (low expression), 101-200 (moderate), and 201-300 (high expression) [34].

\section{Statistical analysis}

SPSS software version 22 (IBM Corp, USA) was used for statistical analysis. Pearson's $\chi 2$ and Spearman's correlation tests were performed to analyze significance of association and correlation between DCLK1-S protein expression and clinicopathological features. Moreover, Kruskal-Wallis and Mann-Whitney $U$ tests were carry out to compare between the groups. DSS and PFS analysis were used to adopting the Kaplan-Meier method, with along the log-rank test to estimate curves between the groups with $95 \%$ confidence interval (Cl). To determination the variables were influenced DSS or PFS, Cox (Proportional Hazards) Regression was performed. All the categorical data were described by $\mathrm{N}(\%)$, a valid percentage, and quantitative data were exhibited as mean, standard deviation (SD), median, and quartile (Q1, Q3). A P-value of $<0.05$ was considered as statistically significant.

\section{Results}

\section{Production of Antibody and Characterization}

\subsection{Production of Anti-DCLK1-S Antibody}


Efficacy of conjugation and immunization was evaluated by SDS-PAGE analysis of the BSA -conjugate and ELISA test, respectively. Smear pattern of SDS-PAGE and the absence of free peptide showed effective conjugation of BSA-peptide (supplementary Fig.1 (A)).

Immunoreactivity of the antibodies was confirmed by ELISA test. The results showed high immunoreactivity of the antibodies reaching to plateau as low as an antibody concentration of $1.25 \mathrm{ng} / \mathrm{ml}$ (supplementary Fig.1 (B)).

\subsection{Validation of Anti-DCLK1-S Antibody}

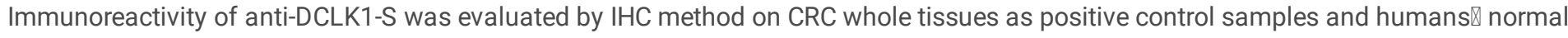
and cancerous tissues of testis, ovary, and skin as negative control samples. In addition, isotype control and primary antibody saturated with immunizing peptide were used as negative reagent controls in all the experiments. The results clearly showed specific pattern of immunoreactivity in CRC tissues compared to negative control tissues (Fig. 2 and supplementary Fig. 2). The antibody could strongly recognize DCLK1-S in CRC samples, and localization was restricted to cytoplasmic area and partially in cell membrane and nucleus area of CRC samples, (Fig. 1). No staining was observed in humans $\$ normal and cancerous tissues of testis, ovary, and skin, demonstrating specific immunoreactivity of the produced anti-DCLK1-S antibody (supplementary Fig. 2).

The commercial anti-DCLK1 antibody, which can recognize C-terminal end of both long and short isoforms of DCLK1 (anti-DCLK1-L/S antibody; ab31704) was used to compare expression pattern of DCLK1-L/S vs. DCLK1-S. Results showed no difference in pattern of expression using the generated anti-DCLK1-S antibody and the commercial anti-DCLK1-L/S antibody, as shown in supplementary Fig.3. No staining was also observed in negative control sections incubated by pre-adsorbed or substituted by pre-immune-purified rabbit IgG as primary antibody (Fig.2).

\section{Immunohistochemical Evaluation of Anti-DCLK1-S Antibody in CRC Tissues}

\subsection{Study Population}

Considering technical problems during TMA construction and IHC staining, among all 385 collected CRC samples and 70 adjacent normal tissues, $348 \mathrm{CRC}$ and 51 adjacent normal tissue samples remained for statistics analysis of DCLK1-S expression. Patients had a mean age of $60 \pm 14.4$ years old, and males accounting for $51.8 \%$ of our study population. Mean tumor size was equal to $5 \pm 2.2 \mathrm{~cm}$ (ranged from 2 to $14 \mathrm{~cm}$ ), of which $236(67.6 \%)$ were less than $5 \mathrm{~cm}$. Regarding available tumor differentiation data of 346 patients; $121(35 \%)$ patients had well, 192(55.5\%) patients had moderate, and 33(9.5\%) patients had poor tumor differentiation. Regarding available data of TNM stage for 296 samples, 52 (17.6\%) cases had stage I, 133 (44.9\%) cases had stage II, 100 (33.8\%) cases had stage III, and 11 (3.7\%) cases had stage IV. Vascular, neural, and lymph node involvement was found in 59 (17.2\%), 67 (19.5\%), and 118 (34.3\%) of cases, respectively. Table 1 summarizes all the clinicopathological characteristics of the study population.

\subsection{Expression of DCLK1-S in the Patients with CRC and Adjacent Normal Samples}

Results of IHC analysis showed that majority of CRC samples $(293 / 348,84.2 \%)$ had a positive DCLK1-S expression. All the positive

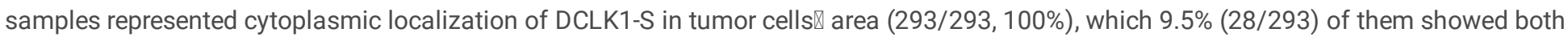
cytoplasmic and nuclear expressions and 4.7\% (14/293) of them showed both cytoplasmic and membranous expression of DCLK1-S (Fig. 1). In terms of intensity, among 348 CRC samples, 36 (10.3\%) of them had strong, 77 (22.2\%) of them had moderate, $180(51.7 \%)$ of them had weak, and 55 (15.8\%) of them had negative staining (Fig. 2). Hscore was classified as low (100 $\leq$ ), moderate (100-200), and high $(201 \geq)$ ) histochemical reactivity. Among 348 cases, 255 (73.3\%) of them showed low, 83 (23.8\%) of them showed moderate, and $10(2.9 \%)$ of them had high immunoreactivity of DCLK1-S. There was a statistically significant difference in expression of DCLK1-S (in terms of intensity of staining, percentage of positive cells, and $H$-score; $\mathrm{P}<0.001)$ between $\mathrm{CRC}$ and adjacent normal samples. The highest expression of DCLK1-S was found only in CRC samples compared to adjacent normal tissue samples (Fig. 3). In terms of intensity, none of the adjacent normal tissues demonstrated strong reactivity of DCLK1-S, but moderate, weak, and negative intensity was found in 3 (5.9\%), $20(39.2 \%)$, and 28 (54.9\%) of tissues. Moreover, in terms of Hscore, 48/51 (94.1\%) of samples displayed low scoring pattern, only $3 / 51$ $(5.9 \%)$ of cases showed a moderate score of DCLK1-S expression, and no high scoring pattern was detected in adjacent normal tissues (Table 2).

\subsection{Cytoplasmic Expression of DCLK1-S Was Associated with Tumor Aggressiveness in CRC Tissues}

The results of Pearson's $\chi 2$ test revealed a statistically significant association between overexpression of DCLK1-S and advanced TNM stage $(P<0.001)$ as well as the increased tumor differentiation $(P=0.03)$. Furthermore, the results of Spearman's correlation test exhibited a direct significant correlation between cytoplasmic DCLK1-S expression and TNM stage $(P<0.001)$ and also tumor differentiation $(P=0.02)$. Moreover, the results of the Kruskal-Wallis test indicated a statistically significant difference between median cytoplasmic expression level 
of DCLK1-S and tumor differentiation ( $P=0.04)$. Mann-Whitney Utest also showed a statistically significant difference in median expression level of cytoplasmic DCLK1-S between poor and moderate tumor differentiation ( $P=0.04)$. Results of statistical analysis did not show any significant association between DCLK1-S expression and other clinicopathological characteristics including age, gender, tumor size, vascular and neural involvement, distant metastasis, and tumor recurrence. All findings are available in Table 1.

\subsection{Cytoplasmic Expression of DCLK1-S Was a Poor Prognostic Factor of Disease-Specific Survival (DSS) in CRC Tissues}

Among 348 CRC samples included in this study, 235 (67.5\%) samples had a history of tumor recurrence, distant metastasis, or cancerrelated death events. Distant metastasis and tumor recurrence occurred in $67(28.5 \%)$ and $72(30.6 \%)$ of patients, respectively, while in 80 $(34 \%)$ of patients, metastasis and recurrence was not observed. During the follow-up period, cancer-related death and the other causes of death were documented in $67(90.5 \%)$ and 7 patients (9.5\%), respectively. Mean and median follow-up times were equal to $43.5(S D=29.7)$ and $38(21,38)$ months, with a range of $1-108$ months.

The results of Kaplan-Meier survival analysis represented that the CRC patients with high cytoplasmic expression of DCLK1-S had shorter DSS compared to those with moderate and low cytoplasmic DCLK1-S expression (log-rank test, $P=0.03)$ ( Fig.4(a)). Mean \pm SD of DSS time was equal to $48 \pm 14,69 \pm 6$, and $81 \pm 3.5$, for the patients with high, moderate, and low cytoplasmic DCLK1-S expression, respectively. There was no significant difference between PFS and the patients with high, moderate, and low cytoplasmic expression of DCLK1-S (logrank test, $P=0.17$ ) (Fig.4 (b)). Mean \pm SD of PFS time was equal to $48 \pm 14,67.7 \pm 6$, and $74 \pm 3.6$, for the patients with high, moderate, and low cytoplasmic DCLK1-S expression, respectively.

Moreover, 5 -year DSS was obtained as 50,62 , and $74 \%$, for the patients with high, moderate, and low level of cytoplasmic expression of DCLK1-S , respectively (mean \pm SD of DSS time was equal to $48 \pm 14,69 \pm 6$, and $81 \pm 3.5$, for the patients with high, moderate, and low cytoplasmic expression of DCLK1-S, respectively $P=0.01$ ). As demonstrated in Table 3 , the results of univariate analysis showed that cytoplasmic expression of DCLK1-S $(P=0.04)$, age $(P=0.04)$, tumor differentiation $(P=0.01)$, TNM stage $(P=0.02)$, vascular invasion $(P=$ $0.02)$, neural invasion $(P=0.03)$, and lymph node involvement $(P=0.001)$ were significant risk factors influencing $D S S$ as confirmed by univariate Cox regression analysis. Moreover, prognostic value was increased in high cytoplasmic expression of DCLK1-S vs. moderate expression of DCLK1-S (HR: 2.70, 95\% Cl: 0.98-7.38; $p=0.04$ ) in the patients with CRC in multivariate analysis. Therefore, high cytoplasmic expression of DCLK1-S compared to moderate expression could be considered as an independent prognostic factor of DSS in multivariate analysis.

\section{Discussion}

DCLK1, a putative CSC marker is a member of microtubule-associated proteins whose biological function has been identified in different human cancers, particularly in colorectal and pancreatic carcinomas $[8,10,35]$. The previous studies have also reported that suppressing DCLK1 had a significant direct correlation with tumor invasiveness and drug resistance in renal clear cell cancer and pancreatic cancer [36, 37]. ). In CRC, upregulation of DCLK1 is significantly associated with tumor progression, aggressiveness, metastasis, and poor prognosis [38]. Therefore, oncogenic role of DCLK1 in a wide range of biological studies has suggested prominence of the DCLK1 marker as a promising molecular target in treatment of CRC. From this point, finding distinguished CSC markers which have not been identified on normal stem cells play a fundamental role in targeted-therapy strategies and tumor eradication. Nakanishi et al., revealed CSC characteristics of DCLK1 in intestinal tumor cells but not in normal stem cells in-vivo [7]. Vreugdenhil et al., cloned different transcripts of DCLK1 and discovered two distinguishing alternative splicing variants of DCLK1 transcripts; DCLK-short and DCLK-long [39]. The previous studies have also highlighted isoform-specific function and localization profile of these transcripts in Wistar rats' brains [12]. Interestingly, epigenetic differences have discovered that DCLK1-S isoform ( $47 \mathrm{KDa})$, originated from $\beta$-promoter plays a significant role during colon carcinogenesis by the response to oncogenic pathways, while DCLK1-L isoform ( 82 KDa) could be detected in the early stage of CRC and normal colonic mucosal samples vs. high-stage tumors $[9,15]$. These investigations have strongly supported that DCLK1-S as an isoform of DCLK1 is identified as a requisite factor in tumor invasion, proliferation, and metastasis potential of CRC cells [15].

Although, many preliminary studies have confirmed clinical value of DCLK1 expression in tumor progression and aggressiveness, the available commercial anti-DCLK1 antibodies widely used in the previous studies could detect sequence homology epitopes of both isoforms, DCLK1-L and DCLK1-S, or only DCLK1-L isoform; hence, specific implication of DCLK1-S has still remained largely unknown.

In our previous works, oncopathological value of DCLK1 expression was evaluated in various cancers including colorectal, bladder, and gastric carcinomas; results of which were in line with other studies [17, 19, 36, 40-43]. In a comprehensive study on 472 bladder cancer cases, an increase was found in expression of DCLK1 (DCLK1-L/S) that associated with more aggressive tumors, advanced stages, and poorer DSS in the patients with bladder cancer [19]. Additionally, results of our previous study on local and circulating DCLK1 (DCLK1-L) in 
58 fresh CRC tissues and their correspondent normal margins demonstrated significantly higher expression of DCLK1 in the patients with CRC having advanced-stage and higher tumor grade in both protein and mRNA expression levels [41]. We have recently reported highly significant expression of DCLK1 (DCLK1-L/S) in CRC cases compared to adenomatous and non-adenomatous colonic polyps, as well as a positive significant correlation between DCLK1 expression and tumor size, tumor differentiation, and lymph node involvement [20]. Other clinical investigations have also represented that higher expression of DCLK1 (DCLK1-L/S) had a significant positive correlation with tumor invasion, metastasis, and shorter survival in the patients with renal clear cell carcinoma and bladder cancer [36, 43]. However, it is important to state that the commercial anti-DCLK1 antibodies used in these studies can only recognize C-terminal end of DCLK1-L/S protein, or only N-terminal end of DCLK1-L isoform; thus, further studies are required to investigate biological role of DCLK1-S in tumor progression and invasiveness.

Since, the absence of the commercial anti-DCLK1-S antibody was the primary obstacle for assessing this molecule at the protein level, in the present study; a polyclonal specific antibody against DCLK1-S was successfully developed in our laboratory. This will provide further opportunities to investigators to obtain more accurate and valuable findings related to DCLK1-S localization and its clinical significance as the unique DCLK1 isoform responsible for invasiveness and aggressive behavior in various tumors by IHC method.

Our findings demonstrated that majority of CRC samples with positive expression of DCLK1-S had pronounced cytoplasmic localization, besides partially nuclear and very low membranous expression patterns of S-isoform. In parallel, the results of immune electron microscopy (IEM) have confirmed the presence of S-isoform in cytosol, mitochondrial, and cell membrane fractions of HCT-116 and COLO205-S-GFP cell lines, unlike L-isoform that was present mainly in cell membrane fractions of HEK293 cells only expressing L-isoform [10]. Other clinical studies used anti-DCLK1-L/S or anti DCLK1-L antibodies have also reported mainly cytoplasmic and membranous localization of DCLK1 in gastric, colorectal, and bladder carcinoma tissues [18, 19, 33, 43], meanwhile in the current study, nucleus localization of DCLK1-S was shown in tumor cells $₫$ area.

Besides, O'Connell et al., suggested that DCLK1-S was the main DCLK1 isoform expressing in humans》 CRC cell lines vs. DCLK1-L markedly expressing in normal colorectal cell lines thus, DCLK1-S can be used as a promising prognostic and targeted-therapeutic marker in treatment of CRC [9]. ). Other studies have demonstrated higher expression of DCLK1-S in polyps from high-risk vs. low-risk patients and also downregulation of DCLK1-L/FOXD3 in polyps from high-risk compared to low-risk patients, indicating the potential suppressor function of FOXD3 in regulation of DCLK1-S [16]. The existence of FOXD3 in normal or low-risk polyps inhibited expression of DCLK1-S, while methylation of FOXD3 in cancerous cells can cause overexpression of DCLK1-S in high-risk cases.

Our comparative immunostaining findings showed similar pattern of immunoreactivity by anti-DCLK1-S antibody and anti DCLK1-L/S antibody, which both represented strong staining of DCLK1 in CRC tissues. Similar staining pattern in both antibodies may be due to the fact that DCLK1-S is predominant isoform in CRC tissues. Although, further studies are required to show different expression of these two isoforms.

The current study provided novel and interesting insights into expression pattern and prognostic significance of DCLK1-S in a well-defined series of CRC tissues. Results of IHC analysis revealed a significant upregulation of cytoplasmic DCLK1-S expression in CRC samples compared to adjacent normal tissues. Furthermore, a significant association was found between high expression of DCLK1-S and advanced TNM stage, the increased tumor differentiation, and poor DSS in these cases. Consistent with our study, there is only one study pointed a significant clinical value of DCLK1-S expression associated with tumor aggressiveness at mRNA level in the patients with CRC [9]. Additionally, our results showed that 5-year DSS was equal to 50,62 , and $74 \%$, for the patients with high, moderate, and low cytoplasmic expression of DCLK1-S, respectively.

\section{Conclusions}

Taken together, for better understanding on specific role of the main DCLK1 isoform in aggressiveness of CRC tumors, herein, a specific polyclonal anti-DCLK1-S antibody was produced identified only by six particular amino acids related to short isoform and its specificity was validated by IHC method in CRC samples. Our findings strongly supported critical role of DCLK1-S as the DCLK1 isoform, which led to tumor aggressiveness and worsened DSS in CRC samples. Importantly, high cytoplasmic expression of DCLK1-S protein compared to moderate expression was considered as an independent poor prognostic factor of DSS in these series of CRC cases. Hence, additional investigations and larger trials on the potential therapeutic role of DCLK1-S as a novel CRC agent can shed new lights on treatment of the patients with CRC.

\section{Abbreviations}


CRC: Colorectal cancer; CSC: Cancer stem cell; DCLK1: doublecortin-like kinase 1; DCLK1-L: DCLK1 long isoform; DCLK1-S: DCLK1 short isoform; FOXD3: Forkhead-Box-D3; FFPE: formalin-fixed, paraffin-embedded; IHC: Immunohistochemistry; TMA: tissue microarray; KLH: Imject Maleimide-activated mckeyhole limpet hemocyanin; BSA: Imject Maleimide-activated bovine serum albumin; ELISA: enzyme-linked immunosorbent assay; SDS-PAGE: sodium dodecyl-sulfate polyacrylamide gel electrophoresis; HRP: horseradish peroxidase; Ig:

immunoglobulin; PBS-T: phosphate buffered saline-Tween20; TMB: 3,3',5,5'-tetramethylbenzidine; TNM: tumor, nodes, and metastases staging; H \& E; hematoxylin \& eosin; H-score: histochemical score; IEM: immune electron microscopy; OD: optical density; HRs: Hazard ratios; Cls: Confidence intervals; PFS: progression-free survival; DSS: Disease-specific survival.

\section{Declarations}

\section{Ethics approval and consent to participate}

The current study was approved by the Human Research Ethics Committee of the Iran University of Medical Sciences, Tehran, Iran (Ref No: IR.IUMS.REC 1396.32186). All the procedures performed in this study were in accordance with the 1964 Declaration of Helsinki and its later amendments. Informed consent was obtained from all individual participants, parents or legally authorized representatives of participants at the time of sample collection with routine consent forms.

\section{Consent for publication}

Not applicable.

\section{Availability of data and materials}

Not applicable.

\section{Competing interests}

The authors declare no competing interests.

\section{Funding}

This study was supported by an Educated Scholar Grant from the National Institute for Medical Research Development (NIMAD) (Grant \#982625). The current study was part of PhD thesis with Grant Number: 96-03-126-32186, which carried out at Oncopathology research center in Iran University of Medical Sciences.

\section{Authors' contributions}

Z.M. and R.Gh. designed and supervised the work; E.K. wrote the manuscript, did literature search, data acquisition and statistical analysis, constructed TMA blocks and performed all experiments. L.S. analyzed interpreted the data, helped to prepare the Tables as well as contributed to edit of the manuscript. M.R. scored TMAs slides after immunohistochemically staining and helped to prepare the Fig. 2. L.E. helped to prepare the blood sampling of the rabbits in animal experiment section. M.R. helped to literature search and prepare the Fig.1. M.A. contributed in manuscript edit, and data analysis. All authors read and approved the final manuscript.

\section{Acknowledgment}

We are grateful of our colleague at Iran University of Medical Sciences who provided insight and expertise that greatly assisted the research. Apart from, it must be declared that the authors received no specific funding for this work.

\section{References}

1. Bray F, Ferlay J, Soerjomataram I, Siegel RL, Torre LA, Jemal A: Global cancer statistics 2018: GLOBOCAN estimates of incidence and mortality worldwide for 36 cancers in 185 countries. CA-CANCER J CLIN. 2018; 68(6):394-424.

2. Sunami E, Tsuno N, Osada T, Saito S, Kitayama J, Tomozawa S, Tsuruo T, Shibata Y, Muto T, Nagawa H: MMP-1 is a prognostic marker for hematogenous metastasis of colorectal cancer. The oncologist. 2000; 5(2):108-114.

3. Kalantari E, Saadi FH, Asgari M, Shariftabrizi A, Roudi R, Madjd Z: Increased expression of ALDH1A1 in prostate cancer is correlated with tumor aggressiveness: a tissue microarray study of Iranian patients. APPL IMMUNOHISTO M M. 2017; 25(8):592-598. 
4. Zolfaghari MA, Karimi A, Kalantari E, Korourian A, Ghanadan A, Kamyab K, Esmaili N, Razavi ANE, Madjd Z: A comparative study of Long Interspersed Element-1 Protein Immunoreactivity in Cutaneous Malignancies. BMC cancer. 2020; $20: 567$.

5. Shi W, Li F, Li S, Wang J, Wang Q, Yan X, Zhang Q, Chai L, Li M: Increased DCLK1 correlates with the malignant status and poor outcome in malignant tumors: a meta-analysis. Oncotarget. 2017; 8(59):100545-100557.

6. Zeki SS, Graham TA, Wright NA: Stem cells and their implications for colorectal cancer. Nature Nat. Rev. Gastroenterol. Hepatol. 2011; 8(2):90-100.

7. Nakanishi Y, Seno H, Fukuoka A, Ueo T, Yamaga Y, Maruno T, Nakanishi N, Kanda K, Komekado H, Kawada M et al: Dclk1 distinguishes between tumor and normal stem cells in the intestine. Nat. Genet. 2013; 45(1):98-103.

8. Westphalen CB, Takemoto Y, Tanaka T, Macchini M, Jiang Z, Renz BW, Chen X, Ormanns S, Nagar K, Tailor Y et al: Dclk1 defines quiescent pancreatic progenitors that promote injury-induced regeneration and tumorigenesis. Cell Stem Cell. 2016 ; 18(4):441-455.

9. O'Connell MR, Sarkar S, Luthra GK, Okugawa Y, Toiyama Y, Gajjar AH, Qiu S, Goel A, Singh P: Epigenetic changes and alternate promoter usage by human colon cancers for expressing DCLK1-isoforms: Clinical Implications. Sci Rep. 2015 ; 5.

10. Sarkar S, Popov VL, O'Connell M, Stevenson HL, Lee BS, Obeid RA, Luthra G, Singh P: A novel antibody against cancer-stem-cell biomarker, DCLK1-S, is potentially useful for assessing colon cancer risk after screening colonoscopy. Lab Invest. 2017; 97(10):12451261.

11. Burgess HA, Reiner O: Altemative splice variants of doublecortin-like kinase are differentially expressed and have different kinase activities. J Biol Chem. 2002; 277(20):17696-17705.

12. Engels BM, Schouten TG, Van Dullemen J, Gosens I, Vreugdenhil E: Functional differences between two DCLK splice variants. Mol. Brain Res. 2004; 120(2):103-114.

13. Bailey JM, Alsina J, Rasheed ZA, McAllister FM, Fu YY, Plentz R, Zhang H, Pasricha PJ, Bardeesy N, Matsui W et al: DCLK1 marks a morphologically distinct subpopulation of cells with stem cell properties in preinvasive pancreatic cancer. Gastroenterol. 2014; 146(1):245-256.

14. Westphalen CB, Asfaha S, Hayakawa Y, Takemoto Y, Lukin DJ, Nuber AH, Brandtner A, Setlik W, Remotti H, Muley A et al: Long-lived intestinal tuft cells serve as colon cancer-initiating cells. J CLIN INVEST. 2014; 124(3):1283-1295.

15. Singh P, O'Connell M, Shubhashish S: Epigenetic regulation of human DCLK-1 gene during coloncarcinogenesis: Clinical and mechanistic implications. Stem Cell Investig. 2016; 2016(SEP).

16. Sarkar S, O'Connell MR, Okugawa Y, Lee BS, Toiyama Y, Kusunoki M, Daboval RD, Goel A, Singh P: FOXD3 Regulates CSC Marker, DCLK1-S, and Invasive Potential: Prognostic Implications in Colon Cancer. Mol Cancer Res. 2017; 15(12):1678-1691.

17. Kalantari E, Asadi Lari MH, Roudi R, Korourian A, Madjd Z: Lgr5 High /DCLK1 High phenotype is more common in early stage and intestinal subtypes of gastric carcinomas. CANCER BIOMARK. 2017; 20(4):563-573.

18. Mirzaei A, Madjd Z, Kadijani AA, Tavakoli-Yaraki M, Modarresi MH, Verdi J, Akbari A, Tavoosidana G: Evaluation of circulating cellular DCLK1 protein, as the most promising colorectal cancer stem cell marker, using immunoassay based methods. CANCER BIOMARK. 2016; 17(3):301-311.

19. Shafiei S, Kalantari E, Saeednejad Zanjani L, Abolhasani M, Asadi Lari MH, Madjd Z: Increased expression of DCLK1, a novel putative CSC maker, is associated with tumor aggressiveness and worse disease-specific survival in patients with bladder carcinomas. Exp. Mol. Pathol. 2019; 108:164-172.

20. Razi S, Sadeghi A, Asadi-Lari Z, Tam KJ, Kalantari E, Madjd Z: DCLK1, a promising colorectal cancer stem cell marker, regulates tumor progression and invasion through miR-137 and miR-15a dependent manner. Clin Exp Med. 2020;1-9.

21. Mirzaei A, Tavoosidana G, Modarressi MH, Rad AA, Fazeli MS, Shirkoohi R, Tavakoli-Yaraki M, Madjd Z: Upregulation of circulating cancer stem cell marker, DCLK1 but not Lgr5, in chemoradiotherapy-treated colorectal cancer patients. Tumor Biol. 2015; 36(6):48014810.

22. Ghods R, Ghahremani MH, Darzi M, Mahmoudi AR, Yeganeh O, Bayat AA, Pasalar P, Jeddi-Tehrani M, Zarnani AH: Immunohistochemical characterization of novel murine monoclonal antibodies against human placenta-specific 1. Biotechnol. Appl. Biochem. 2014; 61(3):363-369.

23. Madjd Z, Durrant LG, Bradley R, Spendlove I, Ellis IO, Pinder SE: Loss of CD55 is associated with aggressive breast tumors. Clin. Cancer Res. 2004; 10(8):2797-2803.

24. Madjd Z, Spendlove I, Moss R, Bevin S, Pinder SE, Watson NF, Ellis I, Durrant LG: Upregulation of MICA on high-grade invasive operable breast carcinoma. archive. Cancer Immunology.2007; 7(1). 
25. Ghods R, Ghahremani M-H, Madjd Z, Asgari M, Abolhasani M, Tavasoli S, Mahmoudi A-R, Darzi M, Pasalar P, Jeddi-Tehrani M: High placenta-specific 1/low prostate-specific antigen expression pattern in high-grade prostate adenocarcinoma. Cancer Immunol. Immunother. 2014; 63(12):1319-1327.

26. Sobin LH, Gospodarowicz MK, Wittekind C: TNM classification of malignant tumours: John Wiley \& Sons; 2011.

27. Grignon DJ: The current classification of urothelial neoplasms. Mod Pathol. 2009; 22(2):S60-S69.

28. Erfani E, Roudi R, Rakhshan A, Sabet MN, Shariftabrizi A, Madjd Z: Comparative expression analysis of putative cancer stem cell markers CD44 and ALDH1A1 in various skin cancer subtypes. TINT J BIOL MARKER. 2016; 31(1):53-61.

29. Kalantari E, Abolhasani M, Roudi R, Farajollahi MM, Farhad S, Madjd Z, Askarian-Amiri S, Mohsenzadegan M: Co-expression of TLR-9 and MMP-13 is associated with the degree of tumour differentiation in prostate cancer. Int. J. Exp. Pathol. 2019; 100(2):123-132.

30. Camp RL, Charette LA, Rimm DL: Validation of tissue microarray technology in breast carcinoma. Lab. Invest. 2000; 80(12):1943.

31. Sadeghi A, Roudi R, Mirzaei A, Zare Mirzaei A, Madjd Z, Abolhasani M: CD44 epithelial isoform inversely associates with invasive characteristics of colorectal cancer. Biomark. Med. 2019; 13(6):419-426.

32. Wu X, Ruan $\mathrm{Y}$, Jiang $\mathrm{H}, \mathrm{Xu} \mathrm{C}$ : MicroRNA-424 inhibits cell migration, invasion, and epithelial mesenchymal transition by downregulating doublecortin-like kinase 1 in ovarian clear cell carcinoma. INT J BIOCHEM CELL B. 2017; 85:66-74.

33. Kalantari E, Asadi Lari MH, Roudi R, Korourian A, Madjd Z: Lgr5High/DCLK1High phenotype is more common in early stage and intestinal subtypes of gastric carcinomas. CANCER BIOMARK. 2017; 20(4):563-573.

34. McCarty Jr K, Miller L, Cox E, Konrath J, McCarty Sr K: Estrogen receptor analyses. Correlation of biochemical and immunohistochemical methods using monoclonal antireceptor antibodies. Arch. Pathol. Lab. Med. 1985; $109(8): 716$.

35. Patel O, Dai W, Mentzel M, Griffin MDW, Serindoux J, Gay Y, Fischer S, Sterle S, Kropp A, Burns CJ et al: Biochemical and Structural Insights into Doublecortin-like Kinase Domain 1. Structure. 2016; 24(9):1550-1561.

36. Weygant N, Qu D, May R, Tierney RM, Berry WL, Zhao L, Agarwal S, Chandrakesan P, Chinthalapally HR, Murphy NT et al: DCLK1 is a broadly dysregulated target against epithelial-mesenchymal transition, focal adhesion, and stemness in clear cell renal carcinoma. Oncotarget. 2015; 6(4):2193-2205.

37. Sureban SM, May R, Lightfoot SA, Hoskins AB, Lerner M, Brackett DJ, Postier RG, Ramanujam R, Mohammed A, Rao CV et al: DCAMKL1 regulates epithelial-mesenchymal transition in human pancreatic cells through a miR-200a-dependent mechanism. Cancer Res. $2011 ; 71(6): 2328-2338$.

38. Gao T, Wang M, Xu L, Wen T, Liu J, An G: DCLK1 is up-regulated and associated with metastasis and prognosis in colorectal cancer. J. Cancer Res. Clin. Oncol. 2016; 142(10):2131-2140.

39. Vreugdenhil E, Engels B, Middelburg R, Van Koningsbruggen S, Knol J, Veldhuisen B, De Kloet ER: Multiple transcripts generated by the DCAMKL gene are expressed in the rat hippocampus. Mol. Brain Res. 2001; 94(1-2):67-74.

40. Meng QB, Yu JC, Kang WM, Ma ZQ, Zhou WX, Li J, Zhou L, Cao ZJ, Tian SB: Expression of doublecortin-like kinase 1 in human gastric cancer and its correlation with prognosis. Acta Academiae Medicinae Sinicae 2013, 35(6):639-644.

41. Mirzaei A, Tavoosidana G, Rad AA, Rezaei F, Tavakoli-Yaraki M, Kadijani AA, Khalili E, Madjd Z: A new insight into cancer stem cell markers: Could local and circulating cancer stem cell markers correlate in colorectal cancer? Tumor Biol. 2016; 37(2):2405-2414.

42. Mohammadi Y, Tavangar SM, Saidijam M, Amini R, Etemadi K, Karimi Dermani F, Najafi R: DCLK1 plays an important role in colorectal cancer tumorgenesis through the regulation of miR-200c. Biomed Pharmacother. 2018; 103:301-307.

43. Zhang S, Zhang G, Guo H: DCAMKL1 is associated with the malignant status and poor outcome in bladder cancer. Tumor Biol. 2017; $39(6)$.

\section{Tables}


Table 1. The association between cytoplasmic DCLK1S expression and clinicopathological parameters of colorectal cancer (CRC) samples (Intensity of staining and H-score)

\begin{tabular}{|c|c|c|c|c|c|c|c|c|c|}
\hline \multirow{3}{*}{$\begin{array}{l}\text { Patients and } \\
\text { tumor } \\
\text { characteristics }\end{array}$} & \multirow{3}{*}{$\begin{array}{l}\text { Total } \\
\text { samples } \\
\text { N (\%) }\end{array}$} & \multicolumn{4}{|c|}{ Intensity of staining N (\%) } & & \multicolumn{3}{|c|}{ H-score N (\%) } \\
\hline & & Negative & Weak & Moderate & Strong & & Low & Moderate & High \\
\hline & & & $(1+)$ & & & $\begin{array}{l}P- \\
\text { value }\end{array}$ & $(0-100)$ & $\begin{array}{l}(100- \\
200)\end{array}$ & $\begin{array}{l}(200- \\
300)\end{array}$ \\
\hline
\end{tabular}

Colorectal

cancer

Mean age,

years (Range)

$\leq$ Mean age

176(52.9) 27(15.3) 85(48.3) 46(26.2) 18(10.2)

120(68.2) 50(28.4) 6(3.4)

$>$ Mean age

157(47.1) 28(17.8)

85(54.1) 26(16.6) 18(11.5) $\quad 0.21$

125(79.6) 28(17.9) $4(2.5)$

0.11

Not identified

Gender

Male

$$
\text { 177(51.8) 29(16.4) }
$$

91(51.4) 35(19.8)

22(12.4) 0.59

130(73.5)

42(23.7)

$5(2.8)$

0.98

Female

$$
\text { 165(48.2) 26(15.8) }
$$
86(52.1) 39(23.6) 14(8.5) 122(73.9) 38(23) 5(3.1)

Mean tumor size $(\mathrm{mm})$

$\leq$ Mean 236(67.6) 31(13.2)

112(32.4) 24(21.4)

125(53.5) 56(23.5)

24(9.8)

54(48.2) 21(18.8)

13(11.6) $\quad 0.20$

169(71.6)

59(25)

8(3.4)

$>$ Mean

Not identified

Differentiation

Well

Moderate

Poor

121(35)

18(14.9)

64(52.9) 26(21.5)

13(10.7)

95(78.5)

20(16.5)

6(5)

192(55.5) 32(16.7) 100(52.1) 40(20.8)

20(10.4) $\quad 0.82$

139(72.4) 50(26) 3(1.6)

0.02

Not identified

Primary tumor

(PT) stage

pT1

$33(9.5)$

$5(15.2)$

$14(42.4)$

11(33.3) 3(9.1)

19(57.6) 13(39.4) 1(3)

\section{pT2}

pT3

PT4

$\begin{array}{llllll}52(17.6) & 9(17.3) & 31(59.7) & 10(19.2) & 2(3.8) & \\ 133(44.9) & 21(15.8) & 73(54.9) & 26(19.5) & 13(9.8) & 0.29 \\ 100(33.8) & 21(21) & 46(46) & 17(17) & 16(16) & \\ 11(3.7) & 1(9.1) & 6(54.5) & 1(9.1) & 3(27.3) & \end{array}$

$\begin{array}{llll}43(82.7) & 8(15.4) & 1(1.9) & \\ 104(78.2) & 27(20.3) & 2(1.5) & <0.001 \\ 73(73) & 23(23) & 4(4) \\ 8(72.7) & 0(0) & 3(27.3) & \end{array}$

Not identified

Vascular

invasion $(\mathrm{VI})$

Present

59(17.2) 14(23.7) 18(30.5) 18(30.5)

9(15.3)

36(61)

21(35.6)

2(3.4)

Absent

284(82.8) $\quad 40(14.1)$

160(56.3) 57(20.1)

27(9.5)

$0.13 \quad 215(75.7)$

61(21.5) 8(2.8)

0.078

Not identified

Lymph node

invasion (LNI)

Involved

118(34.3) 25(21.2)

48(40.7) 27(22.8) 18(15.3)

0.009

$79(66.9)$

$173(76.5)$

33(28)

6(5.1)

0.007

None

$226(65.7) \quad 29(12.8) \quad 130(57.5) \quad 49(21.7) \quad 18(8)$

Not identified 


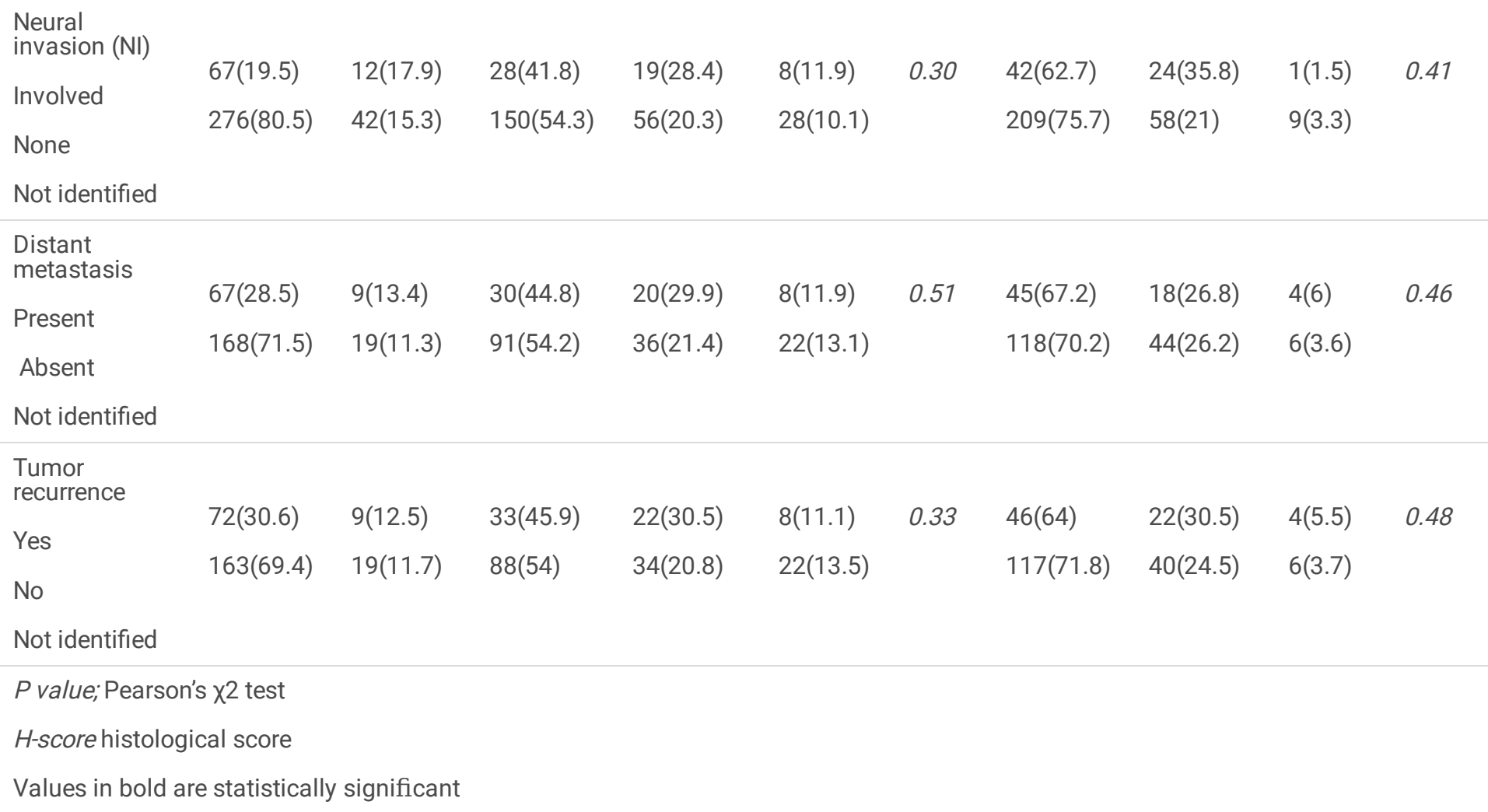




\begin{tabular}{|c|c|c|c|}
\hline Scoring system & CRC samples $\mathrm{N}(\%)$ & Adjacent normal tissue samples $\mathrm{N}(\%)$ & $P$-value \\
\hline \multicolumn{4}{|l|}{ Intensity of staining } \\
\hline Negative (0) & $55(15.8)$ & $28(54.9)$ & \multirow{4}{*}{$<0.001$} \\
\hline Weak $(+1)$ & $180(51.7)$ & $20(39.2)$ & \\
\hline Moderate (+2) & $77(22.2)$ & $3(5.9)$ & \\
\hline Strong $(+3)$ & $36(10.3)$ & $0(0)$ & \\
\hline \multicolumn{4}{|c|}{ Percentage of positive tumor cells } \\
\hline$<25 \%$ & $83(23.9)$ & $33(64.7)$ & \multirow{4}{*}{$<0.001$} \\
\hline $25-50 \%$ & $29(8.3)$ & $2(3.9)$ & \\
\hline $51-75 \%$ & $56(16.1)$ & $4(7.8)$ & \\
\hline$>75 \%$ & $180(51.7)$ & 12(23.6) & \\
\hline \multicolumn{4}{|l|}{ H-score (3 groups) } \\
\hline Low $(0-100)$ & 255(73.3) & $48(94.1)$ & \multirow{4}{*}{$<0.001$} \\
\hline Moderate (101-200) & $83(23.8)$ & $3(5.9)$ & \\
\hline High (201-300) & $10(2.9)$ & $0(0)$ & \\
\hline Total & 348 & 51 & \\
\hline \multicolumn{4}{|c|}{ H-score histological score } \\
\hline \multicolumn{4}{|c|}{$P$ value is based on Mann-Whitney $U$ test } \\
\hline \multicolumn{4}{|c|}{ Values in bold are statistically significant } \\
\hline
\end{tabular}


Table 3. Univariate and multivariate Cox regression analyses of potential prognostic factor for disease-specific survival (DSS) in patients with colorectal cancer (CRC)

\section{Univariate analysis Multivariate analysis}

Covariate

$\begin{array}{lllll} & \text { HR }(95 \% \mathrm{Cl}) & \text { P-value } & \mathrm{HR}(95 \% \mathrm{Cl}) & P \text {-value } \\ \text { Cytoplasmic DCLK1s expression } & & \mathbf{0 . 0 4} & 0.14\end{array}$

High versus Low

1.61(0.97- 2.68)

0.06

1.22(0.67-2.22)

0.51

High versus Moderate

2.58(1.023- 6.52)

0.04

2.70(0.98-7.38)

0.04

Age

1.65(0.79-2.34)

0.04

1.73(1.007-1.99)

0.02

Tumor differentiation

0.01

0.94

Moderate versus well

1.46(0.86- 2.49)

0.15

1.34(0.75-2.38)

0.23

Poor versus well

2.99(1.47- 6.11)

0.003

2.31(0.85-6.31)

0.31

Primary tumor (PT) stage

0.02

0.22

pT2 versus pT1

1.15(0.49- 2.67)

0.74

1.09(0.46-2.57)

0.83

pT3 versus pT1

2.38(1.04- 5.45)

0.04

1.85(0.78-4.38)

0.15

pT4 versus pT1

1.08(0.22- 5.24)

0.91

0.94(0.17-4.98)

0.94

Vascular invasion (VI)

1.84(1.08-3.11)

0.02

Neural invasion (NI)

$0.57(0.34-0.94)$

0.03

Lymph node invasion (LNI)

$0.45(0.28-0.71)$

0.001

Distant metastasis

$0.17(0.10-0.28)$

$<0.001$

Tumor recurrence

$0.18(0.11-0.30)$

$<0.001$

$H R$ hazard ratio, $\mathrm{Cl}$ confidence interval

The variables with $P$ value less than 0.05 and HR more than 1.0 were included in multivariable analyses

Values in bold are statistically significant

\section{Figures}



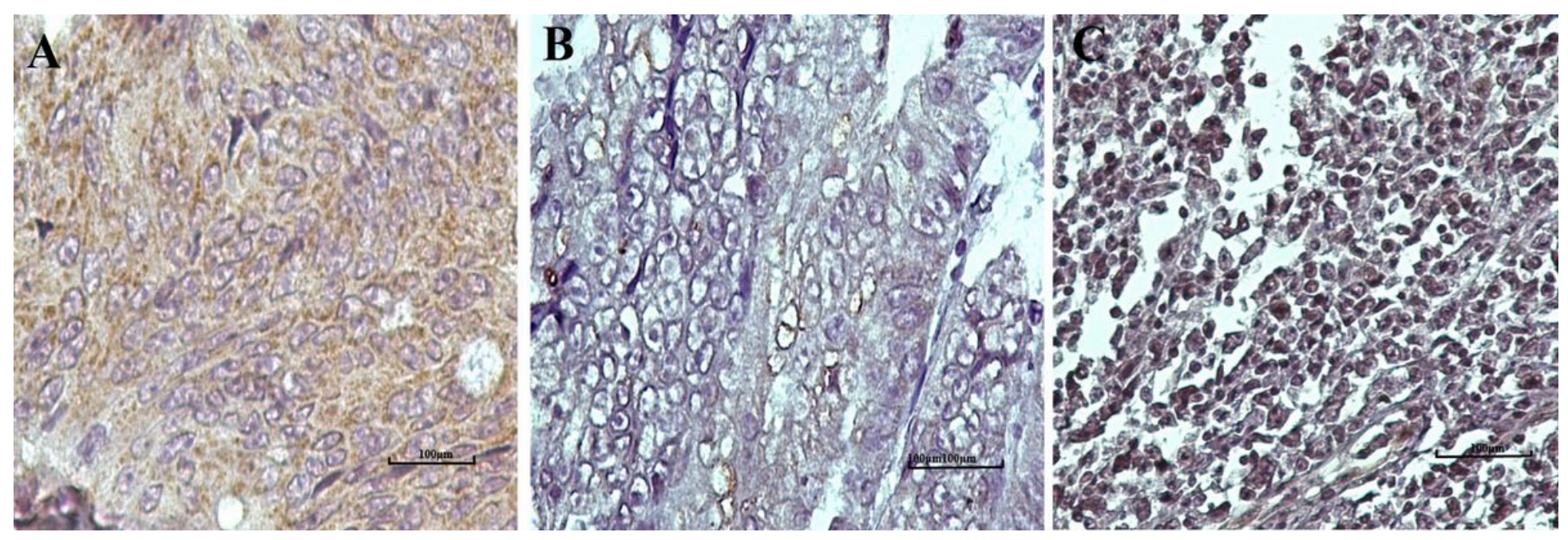

Figure 1

Different localization pattern of DCLK1-S expression in CRC tissues by IHC staining: A) cytoplasmic expression, B) membranous, and C) nucleus expression of DCLK1-S. (All images were taken at $400 \times$ magnification).
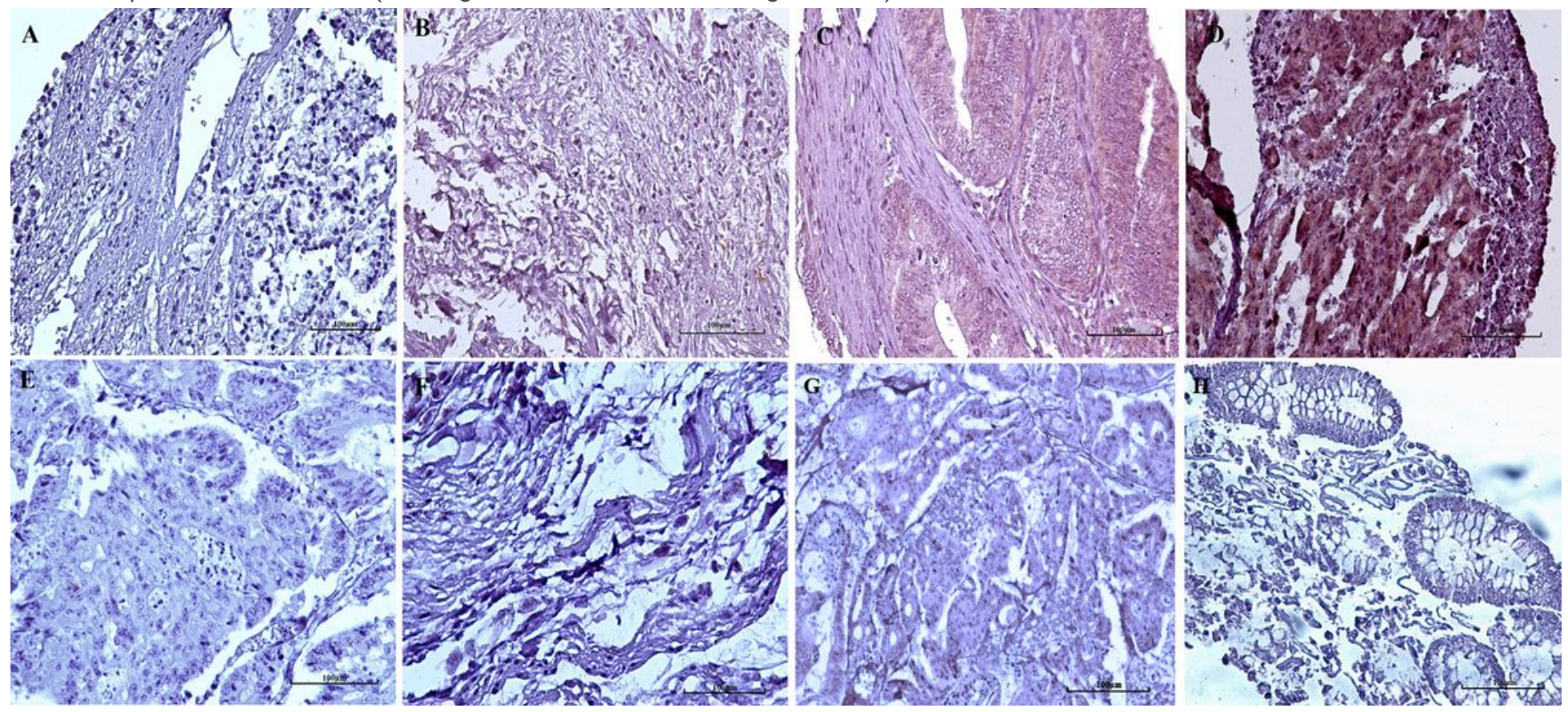

Figure 2

Immunohistochemical staining of DCLK1-S in CRC tissues and negative controles. (A) Negative, (B) low, (C) moderate, and (D) high expression of DCLK1-S in CRC tissue. Replacement of the primary antibodies by (E) pre-immune purified rabbit lgG (isotype control) and (F) Tris Buffer Saline, and (G) primary antibody saturated with immunizing peptide, as the negative reagent controls. $(\mathrm{H})$ Negative expression of DCLK1-S in adjacent normal tissue (All images were taken at 200x magnification). 


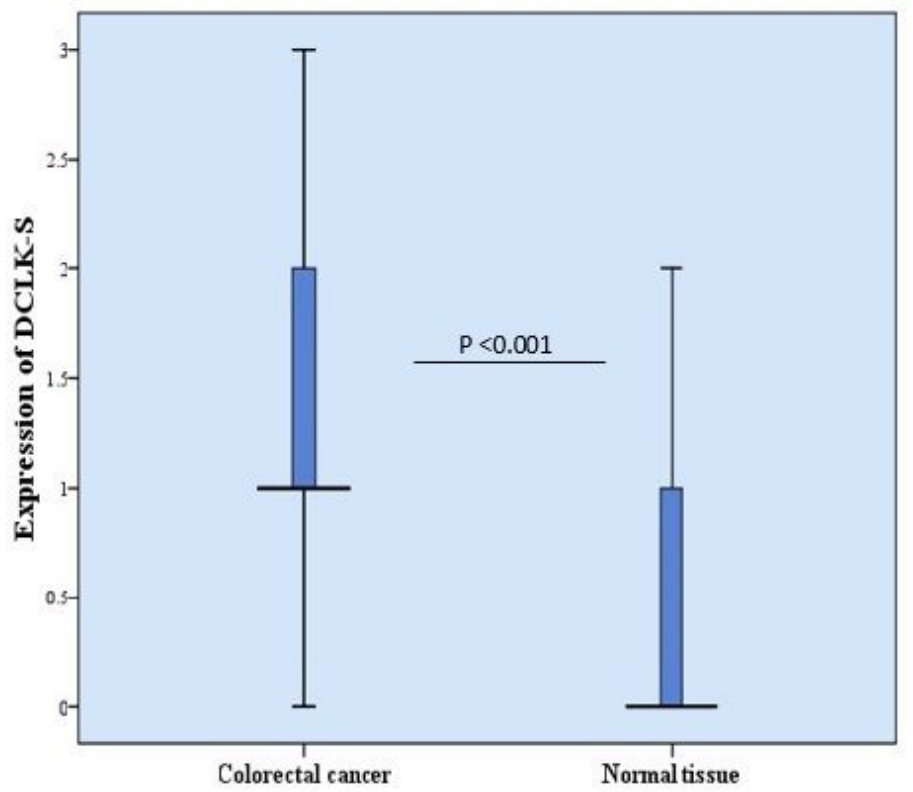

Figure 3

Box plot analysis of DCLK1-S expression levels in CRC specimens versus adjacent normal tissues. The results of Mann-Whitney U test showed that there is a statistically significant differences in the median expression levels of DCLK1-S among CRC and adjacent normal tissues $(P<0.001)$. Based on standard definitions, each box-plot demonstrated the median (bold line) and interquartile lines (box) outliers (circle), and extreme observations (star).
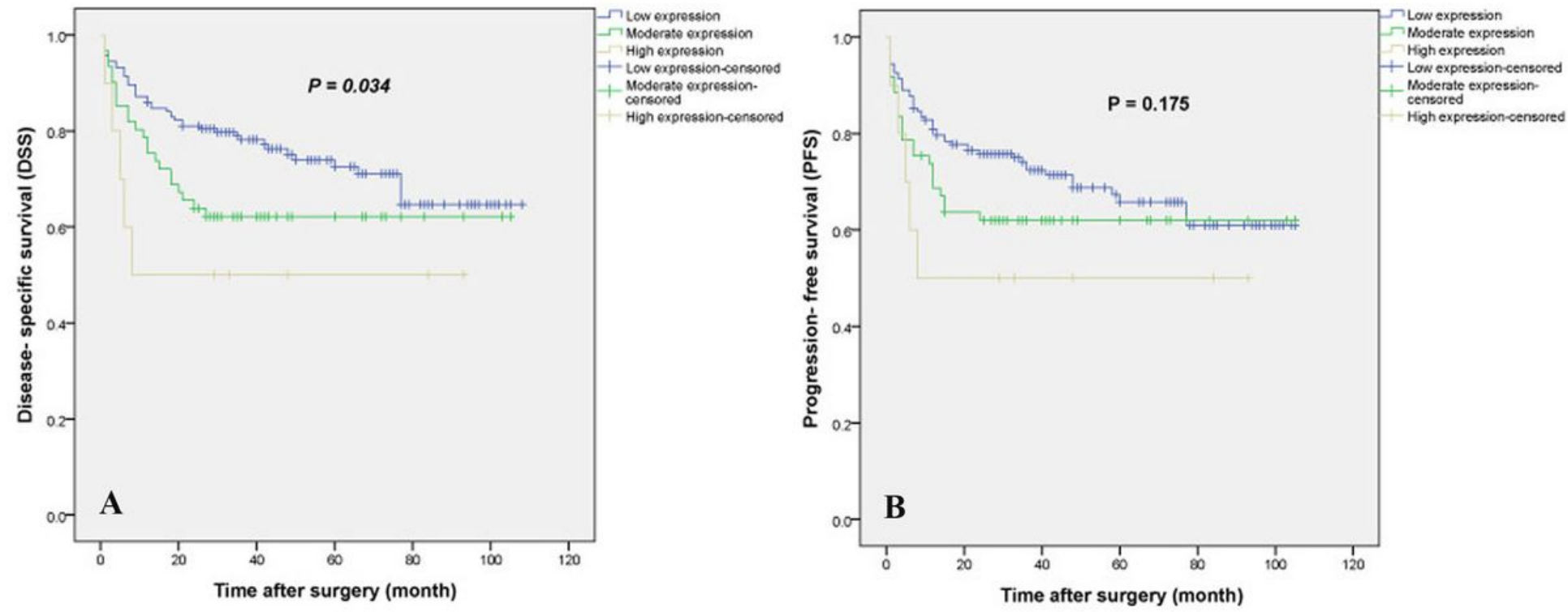

\section{Figure 4}

Kaplan-Meier curves for disease-specific survival (DSS) and progression-free survival (PFS) according to the expression levels of DCLK1-S in CRC. The findings represented (A) there is a significant differences between DSS and the patients with high, moderate and low expression of DCLK1-S $(P=0.034)$ and $(B)$ there was no significant differences between PFS and the patients with high, moderate and low DCLK1-S expression $(P=0.175)$.

\section{Supplementary Files}

This is a list of supplementary files associated with this preprint. Click to download.

- Supplementaryfigures.pptx 